\title{
Análisis métrico de la literatura publicada sobre música Vallenata
}

\author{
Cristina Restrepo-Arango \\ Universidad de Córdoba, División de Bibliotecas y Recursos Educativos, Montería, Colombia \\ crestrepoarango@gmail.com
}

DOI: https://doi.org/10.26512/rici.v12.n2.2019.22048

Recebido/Recibido/Received: 2019-01-16

Aceitado/Aceptado/Accepted: 2019-02-20

Resumen: Analiza la literatura publicada sobre la música vallenata. Para ello se identificó la literatura publicada en la forma de artículos académicos, artículos de difusión, artículos periodísticos, libros, capítulos de libro y ponencias, por medio de las palabras clave "Música Vallenata"; "Vallenato Music"; "Vallenato and Música and Colombia"; "Música and Vallenato and Colombia" en catálogos de bibliotecas colombianas, bases de datos y Google Académico. Los resultados que se obtuvieron fueron exportados a una base de datos bibliográfica en EndNote X5. Los datos obtenidos se analizaron con el software estadístico SPSS para identificar tendencias de publicación, revistas y autores más productivos, crecimiento y colaboración. Se encontraron 276 trabajos publicados desde 1962 hasta diciembre de 2015 por 209 autores, de los cuales se destacan los artículos académicos (54\%) que se han difundido en 108 publicaciones periódicas, libros (24\%) y capítulos de libros (5\%) que se publicaron mayoritariamente en español (97\%). La mayoría de los documentos publicados son en autoría única, solamente $9 \%$ de los trabajos fue publicado en colaboración. Esta literatura creció a una tasa de $8.9 \%$ al año y se duplica aproximadamente cada 19 años.

Palabras clave: Música vallenata. Cienciometría. Bibliometría. Informetría.

Metric analysis of literature published on Vallenata music

Abstract: Analyze the published literature on Vallenata music. For this purpose, the literature published in the form of academic articles, articles of diffusion, journalistic articles, books, book chapters and presentations were identified, by means of the keywords "Music Vallenata"; "Vallenato Music"; "Vallenato and Music and Colombia"; "Music and Vallenato and Colombia" in catalogs of Colombian libraries, databases and Google Scholar. The results that were obtained were exported to a bibliographic database in EndNote X5. The data were analyzed with SPSS statistical software to identify trends in publication, journals and more productive authors, growth and collaboration. We found 276 papers published from 1962 to December 2015 by 209 authors, of which academic articles (54\%) have been published in 108 periodicals, books (24\%) and chapters of books (5\%) Were published mostly in Spanish (97\%). Most of the published documents are in single authorship, only $9 \%$ of the papers were published in collaboration. This literature grew at a rate of $8.9 \%$ per year and doubles approximately every 19 years.

Keywords: Vallenata music. Scientometry. Bibliometrics. Informetrics.

Análise métrica da literatura publicada sobre a música Vallenata

Resumo: Analisa a literatura publicada sobre a música Vallenato. Para este fim, a literatura publicada na forma de artigos acadêmicos, artigos de divulgação, artigos jornalísticos, livros, capítulos de livros e artigos foi identificada por meio das palavras-chave "Música Vallenata"; "Vallenato Music"; "Vallenato e Música e Colômbia"; "Música e Vallenato e Colômbia" em catálogos de bibliotecas colombianas, bases de dados e no Google Scholar. Os resultados obtidos foram exportados para um banco de dados 
bibliográfico no EndNote X5. Os dados obtidos foram analisados com o software estatístico SPSS para identificar as tendências de publicação, periódicos e autores mais produtivos, crescimento e colaboração. Foram encontrados 276 artigos publicados de 1962 a dezembro de 2015, 209 autores, que incluem artigos acadêmicos (54\%) foram distribuídos em 108 periódicos, livros (24\%) e capítulos de livros (5\%) publicados principalmente em espanhol (97\%). A maioria dos documentos publicados é de autoria individual, apenas $9 \%$ dos trabalhos foram publicados em colaboração. Essa literatura cresceu a uma taxa de $8,9 \%$ ao ano e dobra aproximadamente a cada 19 anos.

Palavras-chave: Música Vallenata. Cientometria. Bibliometria. Informetria.

\section{1 - Introducción}

El vallenato es un género musical colombiano que representa la riqueza y la fusión de tres culturas: africana, europea e indígena. Este ritmo musical mezcla las canciones de los vaqueros de la región del Magdalena Grande ${ }^{1}$ en Colombia; los cantos de los esclavos africanos y las danzas de los pueblos indígenas de la Sierra Nevada de Santa Marta (Unesco, 2016). Este género fue influenciado por la poesía española, así como por el uso de instrumentos musicales europeos como el acordeón. También emplea instrumentos musicales autóctonos como la caja de origen africano y la guacharaca de procedencia indígena. Para González (2007, p. 18) “[...] el vallenato es una de las expresiones musicales en las que el legado africano es extraordinariamente rico y claramente visible en la percusión [...]", el vallenato simboliza esa pluriculturalidad que atraviesa a Colombia, pero también esa aculturación que llegó con la conquista, el intercambio comercial y con la incorporación a la vida cotidiana de los medios masivos de comunicación que poco a poco contagiaron y suplantaron objetos, prácticas y por supuesto "contaminaron la pureza" de la música colombiana. Un ejemplo claro de esa aculturación es el uso del "formato de coplas y estribillo, [...que], representa un aporte español relevante [y que es...] uno de los rasgos distintivos de la música vallenata [...]" (GONZÁLEZ, 2007, p. 25-26), estas melodías se fusionaron con ritmos africanos para dar cuenta de lo que sucedía en la cotidianidad de la costa caribe. La música vallenata en sus orígenes más genuinos era un medio de comunicación que usaron los juglares para transmitir oralmente lo que ocurría en las comunidades y viajar de pueblo en pueblo contando historias de forma rítmica y festiva.

El propósito de este trabajo es analizar la literatura publicada sobre la música vallenata con la aplicación de indicadores y modelos cienciométricos para identificar tendencias de publicación, revistas y autores más productivos, crecimiento y colaboración. Para lograr este objetivo se pretende dar respuesta a:

\footnotetext{
${ }^{1}$ Región que incluye los departamentos de Magdalena, Guajira y César
} 
¿Qué tipo de documentos y en cuáles idiomas se publica la literatura sobre la música vallenata?

¿Cuáles son las revistas más productivas que difunden la literatura publicada sobre este asunto?

¿Cuál es la productividad de los autores que publican sobre este tema?

¿Cuál es el índice de colaboración y el grado de colaboración?

¿Cómo crece esta literatura?

Este ritmo musical se convirtió con el paso de los años en insignia de la cultura colombiana en el extranjero, bebido a tres aspectos significativos. Primero, el escritor colombiano Gabriel García Márquez, quien hasta la fecha es el único escritor colombiano en recibir el nobel de literatura, acudió a la entrega de este premio con una agrupación musical vallenata en 1982 en Estocolmo. Además, en su novela "Cien años de soledad" hace alusiones a este género musical, así como en su obra periodística. Segundo, el actor y cantante Carlos Vives grabó e interpretó varios clásicos del vallenato tradicional en una versión contemporánea y su ritmo traspasó las fronteras colombianas. Tercero, la música vallenata tradicional del Caribe colombiano fue inscrita en 2015 en la lista del Patrimonio Cultural Inmaterial de la Unesco (UNESCO, 2016). Estos tres hechos que ocurrieron cronológicamente en momentos diferentes podría suponerse que contribuyeron a la visibilidad de este ritmo musical y que se podría explicar con dos conceptos que son "el principio de jerarquización externa, vigente en las regiones temporalmente dominantes del campo de poder (y también en el campo económico), es decir según el criterio del triunfo temporal calibrado en función de unos índices de éxito comercial [...] o de notoriedad social [..., mientras que el] principio de jerarquización interna, es decir el grado de consagración específica, favorece a los artistas (etc.) que son conocidos y reconocidos por sus partes y sólo por ellos [...]" (BOURDIEU, 2002, p. 322-323). En otras palabras, el principio de jerarquización externo del vallenato ocurrió con el éxito musical que obtuvo Vives, así como con la mención que hizo García Márquez en su obra sobre la música vallenata que ha sido traducida a varios idiomas y el reconocimiento conferido por la UNESCO a este género musical. En el caso del principio de jerarquización interna, se traduce en los premios que recibió Vives, a raíz de que sus canciones comenzaron a ocupar los primeros lugares en listas musicales en varios países.

Estas tres razones son una maravillosa excusa y oportunidad para estudiar este ritmo musical, a partir de la aplicación de indicadores y modelos cienciométricos para conocer tendencias de publicación y otras características de la literatura sobre este asunto. La autora de este trabajo realizó una búsqueda exhaustiva en la literatura para identificar trabajos publicados, cuyo propósito haya sido el estudio métrico de la literatura difundida sobre la 
música vallenata. Sin embargo, no se encontraron estudios con este enfoque, pero se hallaron algunos estudios con otras perspectivas; por ejemplo, la historiografía (Santamaría Delgado, 2009; Posada Consuelo, 2002); la literatura (Pérez Palomino, 2012; Aponte Mantilla, 2011); la etnomusicología (Bermúdez, 2010; González, 2007; Zapata Olivella, 1962), la sociología (SÁNCHEZ CONTRERAS, 2016?) y la lista continúa desde enfoques descriptivos que aglutinan aspectos históricos, etnomusicológicos, sociológicos, etc. (URANGO OSPINA, 2010; CATAÑO BRACHO, 2007; ARAÚJO NOGUERA, 1973). También se revisaron otros trabajos publicados en el ámbito internacional que usaron metrías; por ejemplo, Hancock (2015) que estudió las citas que recibieron los artículos publicados en "Journal of Research in Music Education"; Schubert (2012) que analizó la cooperación entre los músicos de jazz. También Goldenberg (2006) realizó un análisis de contenido de los artículos publicados en "Journal of Music Theory"; y Nelson y Downie (2002) estudiaron una base de datos sobre música con metodologías informétricas. Mientras que, en América Latina, Urbizagástegui Alvarado y Urbizagástegui Alvarado (2012), quienes estudiaron la lírica textual del casete "Martina Portocarrero en Vivo en el Teatro Municipal" aplicando la Ley de Zipf para hallar las palabras clave para la indización.

\section{Marco teórico}

Según González (2007), nadie sabe con quién ni cuándo nació este ritmo musical, quizá la palabra vallenato se convirtió en popular por la bonanza bananera en la región del Magdalena Grande a principios del siglo XX, pues, en ese entonces era común que los trabajadores de las bananeras tocaran instrumentos musicales y cantaran en sus ratos libres, sobre todo porque a principios del siglo XX no había llegado ni la radio ni la televisión al país; por ejemplo, la radio llegó a partir de 1923 y la televisión en la década del 50, es decir, la música se convirtió en una forma de entretenimiento y esto contribuyó a su desarrollo y evolución. Esto coincide con lo que expresó Leandro Díaz en una entrevista a González (2007, p. 70-71) “el vallenato nació como una frase común, una frase callejera, que nadie sabe quién la inventó. Con el asunto del auge del banano, llega mucha gente de esta región a trabajar a la zona bananera y todo el que iba de aquí creían que era del Valle y lo llamaban: 'vallenato'. En ese tiempo se tocaba el acordeón, llevado por la gente de acá y salió la palabra vallenato; pero más era la persona, no la música". Otros autores como Posada Giraldo (2002) consideran que se denominó vallenato, porque nació en el Valle de Upar como se le conocía en la época de la colonia a lo que hoy en día se denomina la ciudad de Valledupar. Sin embargo, el vallenato en la década de los 30 y 40 no se denominaba como lo conocemos hoy en día, pues, algunos testimonios de reconocidos reyes vallenatos; por ejemplo, "Abel Antonio Villa, el primer 
acordeonero que realizó, hacia 1946, una grabación comercial con composiciones personales, aclaró que la música 'que hoy es llamada vallenato', en los treinta y cuarenta era conocida como 'música de acordeón [...Otro rey vallenato,] Andrés Landero, uno de los más importantes compositores sabaneros, [...], explicaba que el término 'vallenato' es nuevo, y agregaba que antes se conocían como 'paseos provincianos' o 'sones magdalenenses' [...]. Finalmente, Pacho Rada, compositor de Plato, Magdalena, y el primer acordeonero que participó, en 1935, en el programa radial 'La Voz de la Patria', Barranquilla, anotó que en su época a esta música vallenata se la llamaba 'parrandera' [...]. En ninguno de esos casos se hablaba, entonces, de 'vallenato', y esta palabra se usaba, en ese momento, como sinónimo del término popular 'caratejo', para llamar a los individuos afectados por la despigmentación de la piel [...]" (POSADA GIRALDO, 2002, p. 3). A partir de lo anterior Posada Giraldo (2002) concluye que el vallenato no un es ritmo musical estrictamente de Valledupar, tal confusión aparece, porque ambas palabras comienzan con el prefijo "valle", pero eso no significa que la música del acordeón sea de exclusividad de esa ciudad colombiana, pues, es un género que surgió en la región del Magdalena Grande que la conforman los departamentos de Cesar, Guajira y Magdalena.

En general, en la literatura revisada sobre la historia del vallenato no hay coincidencias sobre quién, dónde y cuándo nació este género musical. Los autores que esbozan una historia coinciden en que es un ritmo musical que se originó oralmente y que las composiciones eran improvisadas, pues, daban cuenta de las historias que sucedían en la vida cotidiana en ambientes básicamente rurales. Los juglares llevaban su música vallenata viajando de pueblo en pueblo y especialmente en aquellos lugares donde se estaba llevando a cabo alguna festividad. Quizá, ese origen oral dificulta poder documentar su historia, pero lo que es innegable es la riqueza cultural y musical que acompaña al vallenato en su evolución y posicionamiento en Colombia.

¿Por qué el vallenato en sus inicios más remotos fue un género musical estrictamente de la costa caribe? La costa caribe colombiana estaba lo suficientemente lejos del interior del país y por supuesto de la capital, Bogotá, ya que el único medio de comunicación eran los barcos de vapor que transitaban por el río Magdalena, pero estos viajes desde la costa por el río tardaban varias semanas para llegar a Bogotá. Sin embargo, a pesar de la distancia y la falta de una comunicación constante con el interior del país y con otras regiones de Colombia, la costa caribe estaba más cerca por el océano atlántico de otros países caribeños como Cuba, México, Puerto Rico y hasta del continente europeo. También esta cercanía con el mar permitió que a ciudades como Cartagena y Barranquilla llegaran ritmos musicales de otros países por el intercambio comercial y por los visitantes extranjeros que visitaban estas 
ciudades. También esa cercanía facilitó la incorporación de la radio y de la industria fonográfica, que permitió la transformación musical y la difusión del vallenato a otras regiones del país hasta convertirlo en un ritmo musical representativo de la cultura en el ámbito internacional.

Ese intercambio comercial y cultural entre el caribe colombiano con Europa, Cuba, México, etc. permitió una suerte de transformación y evolución del vallenato, pero sobre todo su amplia difusión en la costa y en todo el territorio nacional. Ese intercambio hizo posible tres aspectos. Primero, la organología que empleó el vallenato inicialmente fue de dos guitarras y maracas o guacharaca, pero con el auge de las bananeras a finales del siglo XIX los europeos que llegaron a esa zona de Colombia trajeron el acordeón que modificó las "interpretaciones musicales [...], porque su toque revolucionaba las formas del canto: a diferencia de las flautas, el acordeón daba libertad a la boca para el canto y permitía concentrar en un solo individuo las funciones de cantor e instrumentista" (POSADA GIRALDO, 2002, p. 4). No sólo llegó ese instrumento también se difundieron otros ritmos musicales como valses, polkas, son cubano, etc. que formaron parte del repertorio de las fiestas de la aristocracia costeña, pues, en ese entonces el vallenato era un género musical de una clase rural y campesina que poco tenía que ver con los gustos de esa clase alta costeña. Es así como en la década del 50 se introdujo el acordeón que se utiliza actualmente por las agrupaciones vallenatas. Segundo, el inició de la radio en Colombia trajo la creación de emisoras que transmitían programas culturales y educativos; por ejemplo, los programas de radio teatro que transmitían presentaciones en vivo y en directo de agrupaciones vallenatas y es así como este tipo de iniciativas contribuyó a la consolidación, evolución, modernización y transición del vallenato del ámbito rural al urbano. Tercero, la introducción de la industria fonográfica posibilitó que, en la década del 1940, la música vallenata se grabara en esa tecnología y la incorporación del contrabajo a la organología, lo que ayudó a su difusión, además a que hoy en día los amantes de este género tengan noticia de cantantes vallenatos como Guillermo Buitrago, Leandro Díaz, Rafael Escalona, etc. (GONZÁLEZ, 2007).

¿Qué hizo posible esa aceptación social del vallenato en las élites colombianas? Según González (2007), este género musical se introdujo inicialmente en las clases sociales pobres y posteriormente poco a poco fue escalando posiciones en las distintas clases hasta llegar a las élites regionales, quienes se han apropiado de este género para usarlo con tintes de discurso nacionalista y fundamentalmente enaltecer el pueblo costeño, por medio de la música con fines políticos, pues, es importante recordar que el Cesar hasta 1967 formaba parte de la provincia de Padilla, pero a partir de ese año se fundó el Departamento del Cesar y su primer gobernador fue Alfonso López Michelsen, quien fue nombrado por decreto y quien años más 
tarde sería presidente de Colombia. También fue conocido su fervor y admiración por la música vallenata, tanto así que el lugar donde año tras año se celebra el Festival Vallenato se lleva a cabo en la plaza que tiene su nombre, aunque López Michelsen nació y creció en Bogotá, pero por los avatares políticos fue un defensor acérrimo de la cultura costeña, sobre todo del vallenato.

Otro aspecto que contribuyó a la aceptación social de este género musical es que en las primeras décadas del siglo XX aparecieron trovadores "[...] muy talentosos, que pertenecían a la élite de la zona, que con el correr de los años llegarían a ser considerados paradigmas de esta música. El primero de ellos fue el hacendado Tobías Enrique Pumarejo, nacido en 1906. A diferencia de los trovadores y juglares tradicionales de la época, Pumarejo tuvo acceso a la educación secundaria -un alto nivel en ese tiempo- pues su familia, siguiendo la usanza de las familias adineradas, lo había enviado a realizar tales estudios a Medellín. Las experiencias sonoras y las del entorno social suyo distaban, en mucho, de las de los juglares silvestres como se le conoció- desde sus tiempos estudiantiles" (GONZÁLEZ, 2007, p. 102). La diferencia entre los juglares provenientes de clases sociales no adineradas justamente eran las historias que convertían en letras de canciones las narraciones que contaban su cotidianidad, mientras que los juglares de clases pudientes usaban temas "sofisticados" que seguramente escuchaban en otros ritmos musicales como el bolero, el son cubano, etc., es decir, sus composiciones musicales eran más cercanas a la cotidianidad y gustos de la gente de esas élites. Es de recordar que esos grupos sociales adinerados eran quienes tenían acceso a la tecnología de la época como la radio, la televisión y el disco de acetato, etc. que seguramente también ayudó a ese posicionamiento dentro de esos grupos sociales.

Sin embargo, a pesar de que los medios de comunicación masivos contribuyeron en gran medida a la consolidación del vallenato, para González (2007, p. 150) también esa difusión "[...] ha provocado un desmejoramiento de la calidad de la expresión tradicional -en lo literario y en lo musical- al imponer clichés y estereotipos desprovistos de cualquier interés estético o musicológico. Los mercaderes de la industria fonográfica hacen primar el lucro antes que el valor artístico y por ello privilegian la pequeña inversión que produzca altos dividendos [...]". Esto se evidencia con el pasar de los años; por ejemplo, al escuchar las canciones interpretadas por Guillermo Buitrago, Leandro Díaz, Alejandro Durán, Rafael Escalona, etc. y compararlas con intérpretes actuales como Jorge Celedón, Silvestre Dangond, Juan Carlos Centeno, Carlos Vives, etc. se notan considerablemente las diferencias musicales en las letras de las canciones, la organología que usan y como acompañan las letras, pues, González (2007, p. 150) señala que "[...] cada nueva canción publicada es la repetición de una anterior, con un nuevo texto banal y sensiblero que ha sido encargado por la casa disquera, montada y cortada 
sobre el mismo molde musical que repite los mismos esquemas ad infinitum". Por estas razones y en vista de que es notable la distancia musical entre el vallenato tradicional del vallenato "comercial" la Unesco lo declaró patrimonio inmaterial de la humanidad, con el fin de ayudar a su preservación.

Esa distancia musical entre el vallenato actual y el tradicional es producto del punto de vista de cantantes, compositores y productores, es decir, los creadores de las letras, interpretaciones y arreglos musicales que están inmersos en un mundo social en el que confluyen una diversidad de fuerzas y actores que en el plano musical al igual que en cualquier tipo de obra cultural "[...] supone tres operaciones tan necesarias y unidas como los tres niveles de la realidad social que aprehenden: en primer lugar, el análisis de la posición del campo literario (etc.) [música vallenata] en el seno del campo e poder, y su evolución en el decurso del tiempo; en segundo lugar, el análisis de la estructura interna del campo literario (etc.) [música vallenata], universo sometido a sus propias leyes de funcionamiento y de transformación, es decir la estructura de las relaciones objetivas entre la posición que en el ocupan individuos o grupos situados en situación de competencia por la legitimidad; por último, el análisis de la génesis de los habitus de los ocupantes de esas posiciones [...]" (BOURDIEU, 2002, p. 318). Sin duda las relaciones entre cada uno de los actores en la música vallenata están dadas por la lógica de las relaciones económicas; por ejemplo, en el caso de la música vallenata las tendencias en relación con las letras, ritmos e interpretaciones están dadas por un orden económico que está dominado por el mercado y que en la mayoría de las ocasiones está bastante alejado de la tradición y sobre todo del gusto de aquellos intelectuales que defienden el vallenato tradicional.

\section{Metodología}

Para identificar la literatura publicada sobre este ritmo musical se usaron la combinación de las palabras clave "Música Vallenata"; "Vallenato Music"; "Vallenato and Música and Colombia"; "Música and Vallenato and Colombia”. La búsqueda se realizó en los títulos, palabras clave y/o asuntos de los catálogos de bibliotecas como: Biblioteca Luis Ángel Arango, Biblioteca General de la Pontificia Universidad Javeriana, Biblioteca Nacional de Colombia y WorldCat. También se efectuó una búsqueda en bases de datos como Grove Music, HAPI, JSTOR, Scopus y Web of Science, pero los resultados que se obtuvieron en esas bases de datos extranjeras fueron muy pocos o produjeron cero registros. También se realizó una búsqueda en repositorios de acceso abierto como Scielo y Redalyc, así como en Google Académico, en esta última fuente fue donde se encontró el mayor número de referencias bibliográficas sobre la música vallenata. 
Se usaron como unidades de análisis los documentos publicados sobre la música vallenata en formato de artículo académico, artículo de difusión ${ }^{2}$, artículo periodístico ${ }^{3}$, libro, capítulo de libro, reseña de libro, ponencia y reporte de investigación ${ }^{4}$. Cabe aclarar que no se incluyeron las tesis presentadas para obtener un título académico, ya que en Colombia no existe un catálogo colectivo de tesis y trabajos de grado que facilite la recuperación de la información bibliográfica, aunque algunas de las instituciones de educación superior en los últimos cinco años, por medio de las bibliotecas han dispuesto repositorios de acceso abierto que aglutinan las tesis y trabajos de grado, pero el cubrimiento de estos repositorios es apenas de los últimos años y no es posible acceder a los textos completos de tesis y trabajos publicados antes del 2010. Por esa razón se excluyen este tipo de documento producido.

Los resultados que se obtuvieron fueron exportados a una base de datos bibliográfica en EndNote X5, software de administración de bibliografías. Cada documento que se encontró se revisó y leyó cuidadosamente para corroborar que efectivamente tenía relación con el objeto de estudio de este trabajo, al igual que se revisaron las referencias bibliográficas listadas en cada trabajo para verificar la existencia de estudios que no se hubieran hallado en las fuentes que se consultaron en el proceso de búsqueda. Se normalizaron los nombres de los autores, así como las palabras clave de cada documento, con el fin de evitar duplicación de referencias bibliográficas y en el caso de encontrar referencias bibliográficas duplicadas, se procedió a su eliminación.

Para analizar las características demográficas de la literatura recolectada se usó el software estadístico SPSS (versión 20 para Windows en español).

La productividad de los autores se analizó con la aplicación de la ley de Lotka según el modelo de la máxima probabilidad:

$$
C \times \frac{1}{X^{n}}
$$

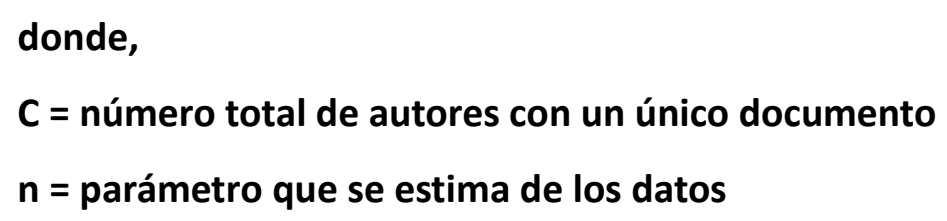

\footnotetext{
${ }^{2}$ Se entiende por artículo de difusión aquellos trabajos breves que explican conceptos, evoluciones, tendencias, etc. que son escritos en un lenguaje que no es especializado y que están destinados a un público general. Son publicados en revistas de difusión como Semana o Credencial, entre otras.

3 Se entiende por artículo periodístico aquel trabajo difundido por medio de periódicos o por los magazines que publican los periódicos en sus ediciones dominicales y que para el caso de la literatura vallenata publicadas son periódicos locales como El Tiempo, El Espectador, etc.

${ }^{4}$ Se entiende por reporte de investigación aquellos trabajos que son producidos como resultado de una investigación, pero no son publicados por los medios formales de publicación, pero son difundidos por la web.
} 
Para medir la colaboración se usó el Índice de Colaboración (IC) propuesto por Lawani (1980) que no es más que el número medio de autores por artículo. Este IC es representado por la siguiente ecuación:

$$
I C=\frac{\sum_{j=1}^{k} j f_{j}}{N}
$$

También se usó Grado de Colaboración (GC) propuesto por Subramanyam (1988), quien afirma que el grado de colaboración en una disciplina se define como la tasa del número de artículos de investigación en colaboración en relación con el número total de artículos de investigación publicados en la disciplina durante un periodo determinado, puede ser expresado como:

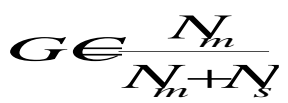

donde,

$\mathrm{GC}=$ Grado de colaboración

$\mathrm{Nm}=$ El número de artículos de investigación de múltiples autores publicados durante determinado año, $y$

Ns = El número de artículos de investigación de autores únicos publicados durante el mismo año.

Para analizar el crecimiento de la literatura producida se usó la función matemática que se representa como:

$C(t)=C(O) e^{a t}$

De acuerdo con Egghe y Ravichandra Rao (1992), esta función puede ser re-escrita como:

$C(t)=c g^{t}$

donde $\mathrm{c}>0, \mathrm{~g}>1, \mathrm{yt} \geq 0$. 
Para hallar el periodo de duplicación, se usará la siguiente ecuación

$$
\begin{aligned}
& n(\log g)=\log 2.0 \\
& n=\frac{\log 2.0}{\log g}
\end{aligned}
$$

El cálculo de los parámetros de la distribución exponencial se realizó por el método de la determinación de la regresión de la curva no lineal. Como se espera una alta correlación entre las variables dependientes e independientes, esa correlación fue explorada usándose el coeficiente de determinación $\mathrm{R}^{2}$ al 0.05 nivel de significancia.

\section{Resultados}

Se encontraron un total de 276 trabajos publicados en diferentes tipos de documentos desde 1962 hasta diciembre de 2015 como se muestran en la Tabla 1.

\begin{tabular}{|c|c|c|c|c|c|c|c|c|c|c|c|c|c|}
\hline \multirow{2}{*}{ Años } & \multicolumn{8}{|c|}{ Español } & \multicolumn{3}{|c|}{ Inglés } & \multirow{2}{*}{$\begin{array}{c}\text { Francés } \\
\mathrm{CL}\end{array}$} & \multirow{2}{*}{ Total } \\
\hline & $\mathbf{L}$ & CL & PON & ART & AM & AP & RES & REPO & $\mathbf{L}$ & ART & $C L$ & & \\
\hline 1962-1969 & -- & -- & -- & 1 & -- & -- & -- & -- & -- & -- & -- & -- & 1 \\
\hline 1970-1975 & 1 & -- & -- & -- & -- & -- & -- & -- & -- & -- & -- & -- & 1 \\
\hline 1976-1980 & 3 & -- & -- & 1 & -- & -- & -- & -- & -- & -- & -- & -- & 4 \\
\hline 1981-1985 & 5 & 1 & -- & 2 & 2 & 4 & -- & -- & -- & -- & -- & -- & 14 \\
\hline 1986-1990 & 7 & -- & -- & 4 & -- & 2 & 2 & -- & -- & -- & -- & -- & 15 \\
\hline 1991-1995 & 4 & -- & -- & 4 & -- & 2 & -- & -- & -- & -- & -- & -- & 10 \\
\hline 1996-2000 & 13 & -- & 1 & 26 & 1 & 4 & 4 & -- & 1 & -- & -- & 1 & 50 \\
\hline 2001-2005 & 13 & 3 & 7 & 19 & 2 & -- & 3 & -- & -- & 1 & 2 & -- & 45 \\
\hline 2006-2010 & 13 & 6 & 2 & 43 & -- & -- & 1 & 1 & -- & 1 & & -- & 67 \\
\hline 2011-2015 & 10 & & 2 & 46 & -- & -- & 4 & 1 & -- & 2 & 1 & -- & 66 \\
\hline Total & 69 & 10 & 9 & 146 & 5 & 12 & 14 & 2 & 1 & 4 & 3 & 1 & 276 \\
\hline
\end{tabular}

Tabla 1. Número de artículos por intervalo de años, idiomas y tipo de documentos

Los tipos de documento que se destacan en la literatura producida sobre el vallenato son los artículos publicados en revistas académicas que suman un total de 150 (54\%), mientras que 73 (24\%) documentos fueron publicados como libros. Le siguen capítulos de libro con 14 
(5\%), reseñas de libros, artículos periodísticos, ponencias y artículos publicados en revistas de difusión no superan 13\% (54 documentos). La tendencia de publicar artículos en revistas académicas sobre este tema tiene relación con la función social del artículo académico que señala Price (1973, p. 117) al afirmar que el origen social del artículo es el "deseo de todo hombre de registrar sus contribuciones y de reservárselas. Sólo incidentalmente el artículo sirve de vehículo de información, de noticia de nuevos conocimientos promulgada en provecho de todos". Sin embargo, no es un asunto simplista el deseo de registrar el conocimiento en un documento para tener la autoría sobre esas ideas y posteriormente ser citado por otros autores, sino como lo afirma Bourdieu (2001, p. 31-32), “...los productores producen allí [en el documento] prioritariamente para otros productores, hay que distinguir el capital necesario para la simple producción de un habla corriente más o menos legítima y el capital de instrumentos de expresión que suponen la apropiación de los recursos depositados objetivamente en las bibliotecas, [como] los libros, y en especial los "clásicos", las gramáticas y los diccionarios [que son] necesarios para la producción de un discurso escrito digno de ser publicado, es decir, oficializado". En otras palabras, el científico necesita tener el dominio de la gramática de un idioma, en este caso del español, para "oficializar" sus ideas, hallazgos y teorías, de esta manera formar parte de ese "capital cultural" de una disciplina y asegurar que no solamente se escribe para la "posteridad", sino para otros investigadores y lectores interesados en el tema.

Estos documentos se publicaron principalmente en el idioma español con 267 trabajos (97\%), mientras que el inglés y el francés apenas representan 9 documentos (3\%). La literatura sobre este asunto es de interés local, aunque a partir del auge del proyecto musical "Clásicos de la Provincia" de Carlos Vives en 1993, que se difundió tanto en ámbito nacional como internacional, lo que contribuyó a incrementar el interés sobre este género por investigadores nacionales y por algunos extranjeros como es el caso del británico Peter Wade, quien es doctor en antropología social, profesor de la Universidad de Manchester y un estudioso de las relaciones étnicas en América Latina, así como el francés Gérard Borras, Peter Thomson y otros. Aunque en la literatura que se reunió para hacer este estudio no se encontró un número significativo de investigadores extranjeros que hayan publicado trabajos sobre la música vallenata, lo que se puede confirmar con la escasa o casi nula presencia de documentos indizados en las bases de datos de "corriente principal" como es el Web of Science y Scopus, así como otras bases de datos especializadas en estudios de América Latina o en música como es el caso de HAPI y Grove Music. Por ejemplo, se encontraron 150 artículos (54\%) publicados sobre el vallenato, pero esas bases de datos no indizan las revistas académicas o revistas de difusión colombianas en las cuales se publicaron esos documentos. A partir de esto es 
importante resaltar la urgente necesidad de construir una base de datos bibliográfica nacional que unifique no sólo los metadatos, sino que incluya la producción bibliográfica nacional en una única herramienta que facilite la recuperación de información y obtención de indicadores cienciométricos para desarrollar la vigilancia científica.

El documento más antiguo publicado sobre vallenato fue escrito por Manuel Zapata Olivella en el año de 1962, éste apareció en el "Boletín Cultural y Bibliográfico" editado por el Banco de la República de Colombia. Este trabajo trata sobre el uso del acordeón en la costa caribe colombiana, específicamente en la música vallenata. Es importante resaltar que este documento se publicó mucho antes de la creación del departamento del Cesar y por lo tanto el artículo se titula "El acordeón en el Magdalena", es decir, Zapata Olivella (1962) se refiere a la presencia del acordeón en la música de la región del Magdalena Grande. Pasaron más de 10 años para que Consuelo Araújo Noguera publicará el libro titulado "Vallenatología: orígenes y fundamentos de la música vallenata" en 1973, libro que contiene aspectos históricos, sociales y etnomusicológicos del origen y evolución del vallenato. A partir del libro de Araújo Noguera los estudios sobre este género musical de la costa caribe colombiana tomaron un auge destacable en el ámbito nacional. Para muchos autores el primer documento publicado sobre la música vallenata es de esta autora; no obstante, el primer documento data de 1962 como se menciona en líneas anteriores.

La Tabla 2 presenta los títulos de revistas que difundieron el mayor número de artículos sobre la música vallenata. Se encontraron 108 títulos de publicaciones periódicas que suman un total de 150 artículos publicados por este importante vehículo de comunicación que son las revistas. Las seis revistas que publicaron más de cuatro artículos son publicaciones nacionales, tres de ellas son publicadas en ciudades de la costa caribe colombiana como son "Aguaita: Revista del Observatorio del Caribe Colombiano", "La Revista del Vallenato", y "Huellas: Revista de la Universidad del Norte". Aunque también sobresale "Boletín Cultural y Bibliográfico" que es editado en Bogotá por el Banco de la República como una de las instituciones que promueve la cultura colombiana en todos los ámbitos y expresiones. Cabe destacar que seis títulos de revistas publicaron 52 artículos (34\%), mientras que 85 títulos de revistas publicaron un único artículo que representa $56 \%$ del total de artículos. 
Tabla 2. Revistas con el mayor número de artículos

\begin{tabular}{|l|c|}
\hline \multicolumn{1}{|c|}{ Título publicación seriada } & No. Artículos \\
\hline Aguaita, Revista del Observatorio del Caribe Colombiano & 14 \\
\hline Boletín Cultural y Bibliográfico & 14 \\
\hline La Revista del vallenato & 12 \\
\hline Huellas: Revista de la Universidad del Norte & 4 \\
\hline Estudios de Literatura Colombiana & 4 \\
\hline Boletín de Antropología & 4 \\
\hline
\end{tabular}

La Tabla 3 presenta la productividad de los autores, según la ley de Lotka por el método de la máxima probabilidad. Se hizo un conteo completo de todos los autores y se obtuvo un valor para $\mathrm{c}=0.783$ con error estándar de $0.011, \mathrm{n}=2.635$ con un error estándar de 0.101 y con un $\mathrm{R}^{2}$ es de 0.998 , es decir, una confiabilidad de $99 \%$. Los 276 documentos publicados sobre la música vallenata fueron producidos por 209 autores, de éstos 79\% publicaron un único trabajo, mientras que 21\% de los autores publicaron entre 2 hasta 12 documentos. Este hallazgo coincide con la afirmación de Ramsden (1994), para quien la tasa de productividad es baja entre los científicos y altamente variable. También Fox (1983, p. 286) sostiene que "la tasa promedio de publicación tiende a ser baja, la variación entre los científicos es muy alta. Si se observa la publicación durante un año, un período de cinco años, o toda la vida profesional, la productividad varía enormemente entre los científicos".

Tabla 3. Productividad de los autores

\begin{tabular}{|c|c|c|}
\hline Contribuciones & Autores & Estimados \\
\hline 1 & 165 & 165,05 \\
\hline 2 & 26 & 24,87 \\
\hline 3 & 5 & 8,22 \\
\hline 4 & 4 & 3,75 \\
\hline 5 & 4 & 2,04 \\
\hline 6 & 2 & 1,24 \\
\hline 7 & 0 & 0,81 \\
\hline 8 & 2 & 0,56 \\
\hline 9 & 0 & 0,41 \\
\hline 10 & 0 & 0,31 \\
\hline 11 & 0 & 0,24 \\
\hline 12 & 1 & 0,19 \\
\hline Total & $\mathbf{2 0 9}$ & $\mathbf{2 0 7 . 6 9}$ \\
\hline
\end{tabular}


La Tabla 4 presenta la estratificación de los autores productores de publicaciones sobre música vallenata, según los criterios sugeridos por Walter, et al. (2010) y adaptados de Braun, Glänzel y Schubert (2001) en categorías que muestran la continuidad o la permanencia en el campo de investigación. Se destacan los autores que se categorizan como One-Timers (91\%), es decir, son los autores que se interesaron por única vez en el tema, ya sea porque fueron alumnos de postgrado y elaboraron un trabajo académico producto de una tesis sobre el tema, pero con la maduración de su carrera académica mudaron a otras temáticas, o bien, son los primeros y únicos trabajos de una gran cantidad de autores. Los autores categorizados como retirantes (4.3\%), son aquellos investigadores que abandonaron el campo o tema de investigación, ya que en los últimos cuatro años no han publicado ningún trabajo sobre el tema. Los productores moderados (4\%) y los grandes productores representados por un único autor $(0.5 \%)$, estas dos categorías en total suman $4.5 \%$ (nueve autores) y concentran 44 (16\%) documentos publicados del total de 276 .

Tabla 4. Categorización estratificada de la productividad de los autores

\begin{tabular}{|l|c|c|}
\hline \multicolumn{1}{|c|}{ Categoría } & No. autores & No. trabajos \\
\hline Grandes productores & 1 & 12 \\
(10 o más trabajos) & $(0.5)$ & $(4.3)$ \\
\hline Productores moderados & 8 & 32 \\
(5 a 9 trabajos) & $(4)$ & $(11.6)$ \\
\hline Retirantes & 9 & 26 \\
(3 a 4 trabajos) & $(4.3)$ & $(9.4)$ \\
\hline One-Timers & 191 & 206 \\
(1 a 2 trabajos) & $(91)$ & $(74.6)$ \\
\hline Total & & $\mathbf{2 7 6}$ \\
\hline
\end{tabular}

Las cifras entre paréntesis son porcentajes

Los moderados y grandes productores están representados por los académicos que se muestran en la Tabla 5. El autor con el mayor número de trabajos publicados es Egberto Bermúdez, quien es músico con estudios de musicología e interpretación de música antigua en el Guildhall School of Music y el King's College de la Universidad de Londres y se desempaña como profesor de la Universidad Nacional de Colombia en el Instituto de Investigaciones Estéticas. Adolfo González Henríquez fue sociólogo y abogado, se desempeñó como profesor de la Universidad del Atlántico. Darío Blanco Arboleda es sociólogo y profesor de la 
Universidad de Antioquia. Marina Quintero es profesora de la Universidad de Antioquia y tiene un programa en la Emisora de esta institución denominado "Una Voz y un Acordeón".

Tabla 5. Autores considerados grandes productores

\begin{tabular}{|l|c|}
\hline \multicolumn{1}{|c|}{ Autor } & No. trabajos \\
\hline Bermúdez, Egberto & 12 \\
\hline González Henríquez, Adolfo & 8 \\
\hline Blanco Arboleda, Darío & 8 \\
\hline Quintero, Marina & 6 \\
\hline Oñate Martínez, Julio Cesar & 6 \\
\hline Urbina Joiro, Hernán & 5 \\
\hline Ochoa Gautier, Ana María & 5 \\
\hline Posada Giraldo, Consuelo & 5 \\
\hline Castillo Mier, Ariel & 5 \\
\hline
\end{tabular}

Julio César Oñate Martínez es compositor, acordeonero, periodista e investigador de la música vallenata. Hernán Urbina Joiro es un escritor y periodista nacido en Valledupar, Colombia. Ana María Ochoa Gautier es profesora en la Universidad de Columbia y estudió el doctorado en etnomusicología y folclore en la Universidad de Indiana. Consuelo Posada Giraldo es profesora de la Universidad de Antioquia. Por último, Ariel Castillo Mier es crítico literario y periodista colombiano. Estos nueve autores tienen en común una nacionalidad, interés por el estudio de la música vallenata que pueden ejercer desde su oficio como académicos, profesores universitarios o periodistas, quienes tienen la embestidura de la autoridad académica para analizar, estudiar y proponer nuevas realidades alrededor de un fenómeno como es el caso de este género musical.

La Tabla 6 presenta el número de firmas por documento discriminado por años. Los documentos publicados en colaboración suman 24 (9\%), mientras que los trabajos con un único autor son 252 (91\%). El primer trabajo publicado en colaboración fue un libro que apareció en 1988 con dos firmas, posteriormente en 1993 se publicó un artículo con 3 firmas y en el año 1994 apareció publicado un libro con dos firmas. La colaboración es entre dos o tres autores, aunque en el año 2009 se publicó un libro con cinco firmas y en el año 2014 se publicó un libro con cuatro firmas. Al parecer la colaboración está ligada con el tipo de documento, pues, justamente apareció con la publicación de un libro y los documentos con el mayor número de firmas son libros. Esto se explica dado que los libros requieren de un mayor esfuerzo, inversión de tiempo y dedicación intelectual de varios investigadores para llevarlo a buen término. También otra razón es que algunos libros son elaborados como compilaciones 
donde participan varios autores con un capítulo de libro que trata sobre un tema previamente seleccionado por los editores del texto. Esta práctica concuerda con los hallazgos de Ossenblok \& Engels (2015) sobre la colaboración en las ciencias sociales y las ciencias humanas, ya que es más frecuente sobre todo en los libros editados por varios autores.

Tabla 6. Número de firmas por documento

\begin{tabular}{|c|c|c|c|c|c|c|}
\hline \multirow{2}{*}{ Años } & \multicolumn{5}{|c|}{ No. Firmas por documento } & \multirow{2}{*}{ Total } \\
\hline & 1 & 2 & 3 & 4 & 5 & \\
\hline 1962-1969 & 1 & -- & -- & -- & -- & 1 \\
\hline 1970-1975 & 1 & -- & -- & -- & -- & 1 \\
\hline $1976-1980$ & 4 & -- & -- & -- & -- & 4 \\
\hline 1981-1985 & 13 & -- & -- & -- & -- & 13 \\
\hline 1986-1990 & 14 & 1 & -- & -- & -- & 15 \\
\hline 1991-1995 & 8 & 1 & 1 & -- & -- & 10 \\
\hline $1996-2000$ & 48 & 4 & -- & -- & -- & 51 \\
\hline 2001-2005 & 44 & 2 & 1 & -- & -- & 47 \\
\hline 2006-2010 & 66 & -- & -- & -- & 1 & 68 \\
\hline 2011-2015 & 53 & 10 & 2 & 1 & -- & 66 \\
\hline Total & 252 & 18 & 4 & 1 & 1 & 276 \\
\hline
\end{tabular}

Desde 1962 hasta 1985 no se publicó ningún trabajo en colaboración, pero es a partir de 1993 que comenzó la publicación en colaboración. Sin embargo, la colaboración para la publicación de trabajos sobre música vallenata presenta fluctuaciones; por ejemplo, el primer trabajo en colaboración apareció en el quinquenio de 1986-1990, mientras que en el quinquenio de 1991-1995 aparecieron publicados dos trabajos en colaboración y en el siguiente quinquenio se duplicó, pero en los dos quinquenios de 2001-2005 y 2006-2010 disminuyó hasta llegar a un único trabajo en colaboración; aunque en el quinquenio de 20112015 se publicaron en colaboración 13 trabajos, es decir, en más de 40 años de publicación sobre este tema se publicaron 24 trabajos en colaboración.

En resumen, la colaboración no es la característica más sobresaliente de la literatura publicada sobre la música vallenata por los resultados que se obtuvieron con la aplicación del índice de colaboración (IC) que se presenta en la Tabla 7. 
Tabla 7. Índice de colaboración por intervalo de años

\begin{tabular}{|c|c|c|c|}
\hline Años & $\begin{array}{c}\text { No. total de } \\
\text { publicaciones }\end{array}$ & $\begin{array}{c}\text { No. total de } \\
\text { publicaciones en } \\
\text { colaboración }\end{array}$ & $\begin{array}{c}\text { Índice de } \\
\text { colaboración }\end{array}$ \\
\hline $1962-1969$ & 1 & -- & -- \\
\hline $1970-1975$ & 1 & - & -- \\
\hline $1976-1980$ & 4 & --- & -- \\
\hline $1981-1985$ & 13 & -- & 6,67 \\
\hline $1986-1990$ & 15 & 1 & 20,00 \\
\hline $1991-1995$ & 10 & 2 & 7,84 \\
\hline $1996-2000$ & 51 & 4 & 6,67 \\
\hline $2001-2005$ & 47 & 3 & 1,49 \\
\hline $2006-2010$ & 68 & 1 & 19,70 \\
\hline $2011-2015$ & 66 & 13 & 62,37 \\
\hline Total & $\mathbf{2 7 6}$ & $\mathbf{2 4}$ & - \\
\hline
\end{tabular}

Quizá la escasa colaboración entre autores que publican sobre este tema hasta el año 2010, se debe a que éstos prefieren la publicación en solitario por el tipo de trabajos que se difunden, pues, son trabajos de reflexión, crítica musical, historia musical, etc., o bien, porque la tendencia en las humanidades es la publicación en solitario, no sólo en los trabajos publicados sobre música vallenata, sino en las humanidades en general. Para Subramanyam (1983) la colaboración varía de una disciplina a otra; por ejemplo, en las ciencias naturales y las ciencias aplicadas los científicos son intensamente colaborativos, principalmente por tres razones. Primero, la disponibilidad de apoyo financiero exige la publicación constante y rápida de resultados de investigación; segundo, los métodos de investigación de las ciencias duras facilitan la colaboración, ya que en la mayoría de los casos se requiere la intervención de varios investigadores de diversas disciplinas; tercero, el ambiente de investigación en las ciencias naturales y las ciencias aplicadas normalmente es en un laboratorio donde se realizan múltiples experimentos. Este panorama difiere enormemente en las ciencias humanas y más específicamente en la música, ya que generalmente la composición y la interpretación de un instrumento es individual, es decir, la colaboración no es una tendencia en esta área del conocimiento.

En síntesis, solamente 24 trabajos (9\%) fueron publicados en colaboración, pero este bajo porcentaje no es una característica sólo de la literatura publicada sobre música vallenta, sino que también en la literatura revisada se encontraron trabajos como el de Ma, Li \& Chen (2014), quienes exploraron la colaboración en las ciencias sociales y humanidades en China, hallaron que la colaboración en disciplinas como arte (14\%), lingüística (24\%), literatura china $(9 \%)$, historia (13\%) y estudios religiosos (11\%), entre otras disciplinas de las humanidades es 
baja comparada con otras disciplinas de las ciencias duras, porque generalmente los artículos son publicados por único autor. También Larivière, Gingras, \& Archambault (2006), analizaron la colaboración en los artículos de autores canadienses indizados en el Web of Science en un periodo de 23 años y encontraron que la colaboración en las humanidades no supera $10 \%$ comparado con $12 \%$ para las ciencias sociales. La literatura sobre música vallenata muestra un comportamiento similar.

La Tabla 8 resume la colaboración en la literatura publicada sobre la música vallenata. El GC corrobora que la colaboración entre autores no es una práctica común en este tema.

Tabla 8. Grado y coeficiente de colaboración

\begin{tabular}{|c|c|c|c|}
\hline Años & $\begin{array}{c}\text { No. de publicaciones } \\
\text { con autores únicos }\end{array}$ & $\begin{array}{c}\text { No. total de } \\
\text { publicaciones en } \\
\text { colaboración }\end{array}$ & $\begin{array}{c}\text { Grado de } \\
\text { colaboración (GC) }\end{array}$ \\
\hline $1962-1969$ & 1 & -- & -- \\
\hline $1970-1975$ & 1 & -- & -- \\
\hline $1976-1980$ & 4 & -- & -- \\
\hline $1981-1985$ & 13 & -- & -- \\
\hline $1986-1990$ & 14 & 1 & 0,07 \\
\hline $1991-1995$ & 8 & 2 & 0,20 \\
\hline $1996-2000$ & 47 & 4 & 0,08 \\
\hline $2001-2005$ & 44 & 3 & 0,07 \\
\hline $2006-2010$ & 67 & 1 & 0,01 \\
\hline $2011-2015$ & 53 & 13 & 0,20 \\
\hline Total & $\mathbf{2 5 2}$ & $\mathbf{2 4}$ & $\mathbf{0 , 6 2}$ \\
\hline
\end{tabular}

La Figura 1 presenta la forma de crecimiento de los documentos producidos sobre la música vallenata. El primer documento publicado es un artículo sobre la influencia y auge del acordeón en el Magdalena Grande en 1962 por Manuel Zapata Olivella. Pasaron más de diez años y se publicó en 1973 el libro de Consuelo Araújo Noguera con el título "Vallenatología: orígenes y fundamentos de la música vallenata". Es a partir de 1973 que comenzó la publicación constante, pero con pocos trabajos publicados sobre la música vallenata en Colombia, pero desde ese año hasta 1996 se publicaron entre 1 a 5 documentos. Si bien es cierto esta realidad cambió en 1997, pues, en este año se triplica la producción con 16 documentos en relación con el año anterior. Seguramente ese ligero incremento tiene relación con la difusión del álbum de Carlos Vives titulado "Clásicos de la Provincia" en 1993 y que tendría un efecto sobre la investigación que se ha producido sobre este género musical cuatro años más tarde, sobre todo porque Vives sacó al vallenato del anonimato local y lo difundió en el plano internacional. 


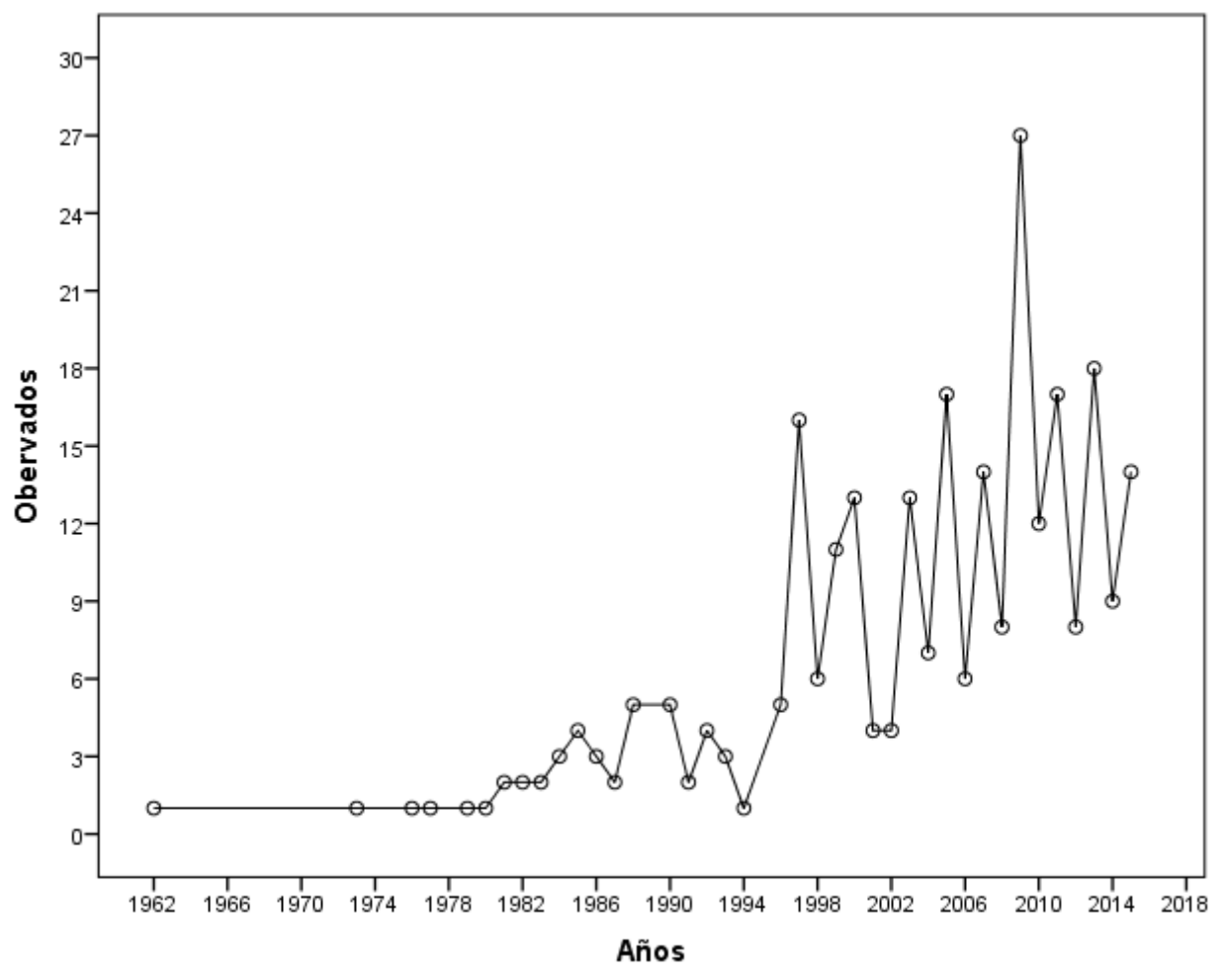

Figura 1. Crecimiento de la literatura sobre la música vallenata

La producción científica sobre el vallenato presenta fluctuaciones desde 1997, pero desde ese año se han publicado más de 4 documentos. Cabe resaltar que el año con el mayor número de documentos producidos fue 2009.

El $R^{2}$ ajustado estimado por el método de la regresión no lineal fue igual a 0.986 . El valor estimado de c fue de 11.705 con un error estándar de 0.935 . El valor estimado de g fue igual a 1.089 y con un error estándar de 0.003. La ecuación que predice el crecimiento exponencial de la literatura sobre la música vallenata es:

$$
C(t)=11.705 \times 1.089^{t}
$$

Esta literatura creció a una tasa de $8.9 \%$ al año. Para estimar el periodo de duplicación se usó la siguiente ecuación:

$$
\text { (1.089) } n=2.0
$$

Tomando los logaritmos de ambos lados de la ecuación se obtiene:

$$
\begin{aligned}
& n(\log 1.089)=\log 2.0 \\
& n=\frac{\log 2.0}{\log 1.089}
\end{aligned}
$$




\section{$n=\frac{0.69315}{0,03702788}$}

\section{$n=18.71$}

Eso significa que las publicaciones se duplican aproximadamente cada 19 años. Según Price (1973), la literatura científica crece exponencialmente y se duplica entre 10 a 15 años. En el caso de la literatura publicada sobre el vallenato se duplica casi en dos décadas, lo que hace pensar que la literatura publicada sobre este tema no ha sido un tema de interés constante para los científicos sociales y humanistas.

La Figura 2 muestra el crecimiento de la literatura publicada sobre la música vallenata.

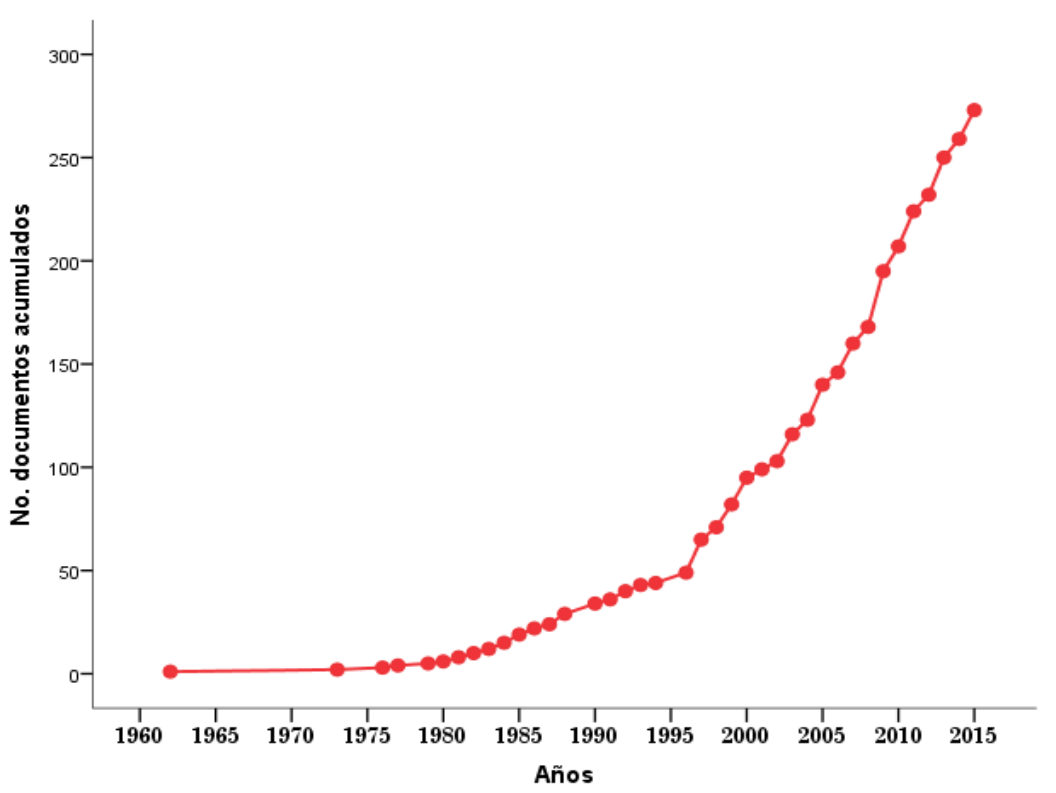

Figura 2. Crecimiento de los documentos según los años

La Figura 3 muestra el crecimiento exponencial de los datos observados y estimados, es decir, las publicaciones sobre este tema aún no están agotadas, pues, seguramente la literatura que se publique continuará incrementándose y traspasando las fronteras nacionales. 


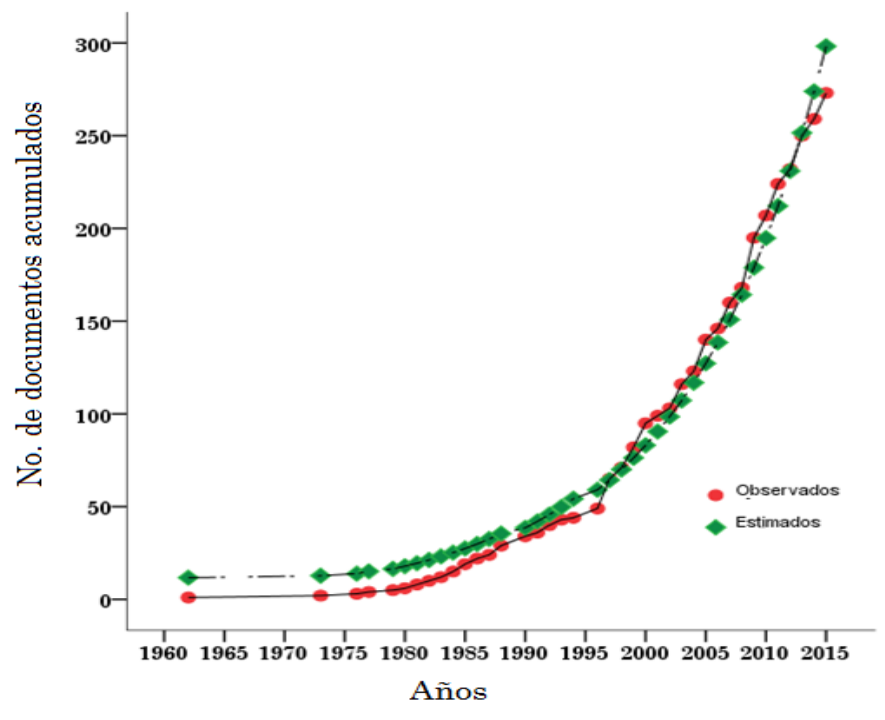

Figura 3. Crecimiento exponencial modelo no-lineal según los años observados y estimados

\section{Conclusiones}

La literatura producida sobre vallenato se publica principalmente en la forma de artículos de revista. Los científicos prefieren difundir sus hallazgos por medio de artículos para comunicarse con sus pares, por medio del uso de artículos publicados en revistas académicas. No se encontraron revistas académicas especializadas indizadas en las bases de datos como Web of Science o Scopus que difundan trabajos sobre la música vallenata, pues, las revistas que canalizan este tipo de información son básicamente revistas locales que se publican en ciudades de la costa caribe colombiana, Bogotá y Medellín, estas publicaciones periódicas no están indizadas en las bases de datos antes mencionadas.

Las revistas más productivas que publican artículos sobre la música vallenata son seis títulos: "Aguaita, Revista del Observatorio del Caribe Colombiano" (14 artículos); "Boletín Cultural y Bibliográfico" (14 artículos); "La Revista del Vallenato" (12 artículos); "Huellas: Revista de la Universidad del Norte"; "Estudios de Literatura Colombiana"; "Estudios de Literatura Colombiana"; y "Boletín de Antropología" (4 artículos cada una). En general, estas revistas publican artículos de orden local y algunas son revistas de difusión como La "Revista del Vallenato".

Entre los autores más productivos sobresale el profesor de la Universidad Nacional de Colombia, Egberto Bermúdez con 12 trabajos, mientras que otros autores como quien fue sociólogo, abogado y profesor de la Universidad del Atlántico, Adolfo González Henríquez con ocho contribuciones; y Darío Blanco Arboleda es sociólogo y profesor de la Universidad de 
Antioquia también con ocho trabajos publicados sobre este tema. En general, la productividad de los autores fue de un único artículo (79\%).

La colaboración entre los autores que publican sobre este tema no está consolidada, a pesar de actualmente la colaboración en la publicación es uno de los aspectos que se está transformando en diversas disciplinas de las ciencias sociales y ciencias humanas. Sin embargo, los datos obtenidos con la aplicación del índice de colaboración y grado de colaboración muestran que solamente 9\% (24 documentos) fueron publicados en colaboración, es decir, este porcentaje está por debajo de lo que obtuvieron otros autores como Larivière, Gingras, \& Archambault (2006) en el área de humanidades donde la colaboración es de $10 \%$. Esta literatura creció a una tasa de $8.9 \%$ al año y se duplica aproximadamente cada 19 años.

Se espera que el auge de Youtube que facilita acceder a diferentes ritmos musicales distintos a los comercialmente difundidos en un país. Es así como Youtube y otras páginas que reúnen videos, películas, documentales y música también facilitan la investigación comparativa para encontrar diferencias o proximidades entre ritmos musicales y más aún del vallenato que es una fusión cultural extremadamente rica para desarrollar investigaciones que ayuden a esclarecer sobre su origen y fortalezcan su preservación.

\section{Bibliografía}

APONTE MANTILLA, María Emilia. La historia del vallenato: discursos hegemónicos y disidentes. Pontificia Universidad Javeriana, Facultad de Ciencias Sociales, Maestría en Literatura, 2011. Trabajo de grado presentado como requisito parcial para optar por el título de Magistra en Literatura.

ARAÚJO NOGUERA, Consuelo. Vallenatología: orígenes y fundamentos de la música vallenata. Bogotá: Tercer Mundo, 1973.

BERMÚDEZ, E. La música colombiana: pasado y presente. En: RECASENS BARBERÀ, Albert; SPENCER ESPINOSA, Christian. A tres bandas: mestizaje, sincretismo e hibridación en el espacio sonoro iberoamericano. Medellín: Museo de Antioquia, 2010. p. 247-255.

BOURDIEU, Pierre. ¿Qué significa hablar? Madrid: Akal Ediciones, 2001.

BOURDIEU, Pierre. Las reglas del arte: génesis y estructura del campo literario. Barcelona: Editorial Anagrama, 2002. p. 318-418.

BRAUN, T.; GLÄNZEL, W.; SCHUBERT, A. Publication and cooperation patterns of the authors of neuroscience journals. Scientometrics, v. 51, n. 3, p. 499-510, 2001.

CATAÑO BRACHO, Juan. El canto vallenato: arte y comunicación. Valledupar: Gráficas del Comercio, 2007.

FOX, Mary Frank. Productivity among scientists: a critical review. Social Studies of Science, v. 13, n. 2, p. 285-305, 1983.

GOLDENBERG, Y. Journal of Music Theory over the years content analysis of the articles and related aspects. Journal of Music Theory, v. 50, n. 1, p. 25-63, 2006. 
GONZÁLEZ, Héctor. Vallenato, tradición y comercio. Santiago de Cali: Universidad del Valle, 2007.

GUPTA, B. M.; SURESH, Kumar; SANGAM, S. L.; KARISIDDAPPA, C. R. Modeling the growth of world social science literature. Scientometrics, v. 53, n. 1, p. 161-164, 2002.

HANCOCK, C. B. Stratification of time to first citation for articles published in the Journal of Research in Music Education: A bibliometric analysis. Journal of Research in Music Education, $\mathrm{v}$. 63, n. 2, p. 238-256, 2015.

LAWANI, S. M. Quality, collaboration and citations in cancer research: a bibliometric study. 1980. Dissertation (Ph.D.) - The Florida State University, 1980.

LARIVIÈRE, V.; GINGRAS, Y.; ARCHAMBAULT, É. Canadian collaboration networks: A comparative analysis of the natural sciences, social sciences and the humanities. Scientometrics, v. 68, n. 3, p. 519-533, 2006.

MA, F.; LI; Y.; CHEN, B. Study of the collaboration in the field of the Chinese humanities and social sciences. Scientometrics, v. 100, n. 2, p. 439-458, 2014.

NELSON, Michael; DOWNIE, J. Stephen. Informetric analysis of a music database. Scientometrics,v. 54, n. 2, p. 243-255, 2002.

OCHOLLA, Dennis N.; ONYANCHA, Omwoyo Bosire. The marginalized knowledge: An informetric analysis of indigenous knowledge publications (1990-2004). SA Journal Libraries \& Information Science, v. 71, n. 3, p. 247-258, 2005.

OSSENBLOK, T. L.; ENGELS, T. C. Edited books in the Social Sciences and Humanities: Characteristics and collaboration analysis. Scientometrics, v. 104, n. 1, p. 219-237, 2015.

PÉREZ PALOMINO, Yineth Paola. Análisis del discurso religioso en las líricas de música de acordeón del Caribe Colombiano entre 1960-1990. Tesis de grado Profesional en Literatura y Lingüística, Universidad de Cartagena, 2012.

POSADA GIRALDO, Consuelo. Canción vallenata: entre la tradición y los intereses comerciales. Estudios de Literatura Colombiana, v. 10, 2002.

Price, Derek J. De Solla. Hacia una ciencia de la ciencia. Estudio preliminar y traducción de José María López Piñero. Barcelona: Editorial Ariel, 1973.

RAMSDEN, Paul. Describing and Explaining Research Productivity. Higher Education, v. 28, n. 2, p. 207-226, 1994.

RAO, I. K Ravichandra; MEERA, B. M. Growth and obsolescence of literature: an empirical study, International Conference on Bibliometrics, Scientometrics, and Informetrics (1991, Bangalore, India), en: Informetrics 91: selected papers from the third International Conference on Informetrics, 9-12 de August, Bangalore: Sarada Ranganathan Endowment for Library Science, 1992. p. 377-394.

SÁNCHEZ CONTRERAS, César Augusto. Función social de la música vallenata. Grupo de Investigación Guatapurí, 2016? Disponible: http://giguatapuri.net/attachments/article/16/FUNCION\%20SOCIAL\%20DE\%20LA\%20MUSICA \%20VALLENATA.pdf Consultado: 4 diciembre 2016.

SANTAMARÍA DELGADO, Carolina. Estado del arte de los inicios de la historiografía de la música popular en Colombia. Memoria y Sociedad, v. 13, n. 26, p. 83-103, 2009.

SUBRAMANYAM, K. Bibliometric studies of research collaboration: a review. Journal of Information Science, v. 6, n. 33, p. 33-38, 1983. 
SCHUBERT, András. Jazz discometrics-A network approach. Journal of informetrics, v. 6, n. 4, p. 480-484., 2012.

UNESCO. El vallenato, música tradicional de la región del Magdalena Grande. Paris: Unesco. Disponible en http://www.unesco.org/culture/ich/es/USL/el-vallenato-musica-tradicional-dela-region-del-magdalena-grande-01095. Consultado 29 de octubre 2016.

URANGO OSPINA, Juan Carlos. Entre lo narrativo y lo descriptivo: ¿Qué predomina en la música de acordeón del Caribe colombiano? Cuadernos de literatura del Caribe $e$ Hispanoamérica, v. 12, p. 169-188, 2010.

URBIZAGÁSTEGUI ALVARADO, Rubén; URBIZAGÁSTEGUI ALVARADO, Flori Martha. Análisis bibliométrico de la lírica textual. El caso de Martina Portocarrero en vivo en el Teatro Municipal. Letras, v. 83, n. 118, p. 153-180, 2012.

WALTER, S. A.; LANZA, B. B. B.; SATO, K. H.; SILVA, E. D. da; BACH, T. M. Análise da produção científica de 1997 a 2009 na área de estratégia: produção e continuidade de atores e cooperação entre instituições brasileiras e estrangeiras. Em: ENCONTRO DA ASSOCIAÇÃO NACIONAL DOS PROGRAMAS DE PÓS-GRADUAÇÃO DE ADMINISTRAÇÃO, 34., 2010, São Paulo. Anais... Rio de Janeiro: ANPAD, 2010.

ZAPATA OLIVELLA, M. Los pasos del folclor colombiano: El acordeón en el Magdalena. Boletín Cultural y Bibliográfico, v. 5, n. 1, p. 81-82, 1962. 